\title{
The development of Leishmania turanica in sand flies and competition with L. major
}

\author{
Alsu Chajbullinova', Jan Votypka ${ }^{1,3^{*}}$, Jovana Sadlova ${ }^{1}$, Katerina Kvapilova' ${ }^{1}$, Veronika Seblova' ${ }^{1}$ Jakub Kreisinger ${ }^{2}$, \\ Milan Jirku ${ }^{3}$, Chizu Sanjoba ${ }^{4}$, Sambuu Gantuya ${ }^{4}$, Yoshitsugu Matsumoto ${ }^{4}$ and Petr Volf ${ }^{1}$
}

\begin{abstract}
Background: In Central Asian foci of zoonotic cutaneous leishmaniases, mixed infections of Leishmania turanica and L. major have been found in a reservoir host (the great gerbil, Rhombomys opimus) as well as in the sand fly vector Phlebotomus papatasi, but hybrids between these two Leishmania species have never been reported. In addition, the role of sand fly species other than P. papatasi in L. turanica circulation is not clear.
\end{abstract}

Methods: In this work we compared the development of $L$. turanica in three sand fly species belonging to different subgenera. In addition, we studied experimental co-infections of sand flies by both Leishmania species using GFP transfected L. turanica (MRHO/MN/08/BZ18(GFP $\left.{ }^{+}\right)$) and RFP transfected L. major (WHOM/IR/-/173-DsRED(RFP $\left.{ }^{+}\right)$). The possibility of Leishmania genetic exchange during the vectorial part of the life cycle was studied using flow cytometry combined with immunofluorescent microscopy.

Results: Late-stage infections of $L$. turanica with frequent colonization of the stomodeal valve were observed in the specific vector P. (Phlebotomus) papatasi and in the permissive vector P. (Adlerius) arabicus. On the other hand, in $P$. sergenti (the specific vector of $L$. tropica), L. turanica promatigotes were present only until the defecation of bloodmeal remnants. In their natural vector P. papatasi, L. turanica and L. major developed similarly, and the spatiotemporal dynamics of localization in the sand fly gut was the same for both leishmania species. Fluorescence microscopy in combination with FACS analyses did not detect any L. major / L. turanica hybrids in the experimental co-infection of $P$. papatasi and P. duboscai.

Conclusion: Our data provide new insight into the development of different leishmania parasite species during a mixed infection in the sand fly gut. Despite the fact that both Leishmania species developed well in P. papatasi and P. duboscai and did not outcompete each other, no genetic exchange was found. However, the ability of L. turanica to establish late-stage infections in these specific vectors of $L$. major suggests that the lipophosphoglycan of this species must be identical or similar to that of L. major.

Keywords: Leishmania turanica, L. major, Mixed infections, Competition, Genetic exchange, Vector competence, Phlebotomus

\section{Background}

Leishmaniases are vector-born diseases transmitted by phlebotomine sand flies and caused by a protozoan flagellate of the genus Leishmania. Leishmania major, the main causative agent of zoonotic cutaneous leishmaniasis (ZCL) is spread over a wide area in Africa, the Middle East and Asia, while L. turanica, a species less

\footnotetext{
* Correspondence: vapid@natur.cuni.cz

'Department of Parasitology, Fac. Sci, Charles University in Prague, Prague, Czech Republic

${ }^{3}$ Biology Centre, Institute of Parasitology, Ceske Budejovice, Czech Republic Full list of author information is available at the end of the article
}

pathogenic to humans [1] is found mainly in Central Asia (reviewed by [2]).

In Central Asia, the great gerbil (Rhombomys opimus) is well documented as a reservoir host for both L. turanica and $L$. major, which may also occur in sympatry with nonpathogenic L. gerbilli (reviewed by [3]). All three Leishmania species have been found to coexist in a single gerbil, and mixed infections of L. turanica and L. major are frequent [4-7]. In sympatric areas, mixed infections of $L$. major and $L$. turanica have a significant effect on the circulation of $L$. major in the wild; co-

\section{() Biomed Central}

(c) 2012 Chajbullinova et al.; licensee BioMed Central Ltd. This is an Open Access article distributed under the terms of the Creative Commons Attribution License (http://creativecommons.org/licenses/by/2.0), which permits unrestricted use, distribution, and reproduction in any medium, provided the original work is properly cited. 
infection with $L$. turanica results in an increased duration of $L$. major infection in gerbil populations and enables the persistence of $L$. major to the subsequent transmission season [5,8]. Phlebotomus papatasi is believed to be the vector for both Leishmania species [3], and mutual circulation in P. papatasi has been recently reported using molecular methods $[9,10]$.

A different situation regarding the transmission cycle has been described in China and Mongolia. In these areas, the presence of $L$. major and its main vector $P$. papatasi has not been demonstrated, and only the circulation of L. turanica and L. gerbilli in R. opimus has been found [1,4,11]. In addition to P. (Phlebotomus) papatasi, L. turanica has been reported from two members of the subgenus $P$. andrejevi and $P$. mongolensis in China [1]. However, the role of these vectors in the circulation of $L$. turanica is not clear.

In the present study, we compared the development of L. turanica in three sand fly species distributed in Central Asia and the Middle East: P. papatasi and P. (Paraphlebotomus) sergenti represent vectors specific for $L$. major and L. tropica (reviewed by [12]), respectively, while Phlebotomus (Adlerius) arabicus was chosen as a member of the permissive vectors susceptible to various Leishmania spp. (reviewed by [13]). Finding that L. turanica and L. major develop similarly and co-localize in P. papatasi midgut led us to attempt to detect possible genetic hybrids using fluorescent marked L. turanica and L. major strains. Genetic exchange in Leishmania is a very rare event (reviewed by [14]) but has important epidemiological consequences; it can rapidly enhance the fitness of the parasite $[15,16]$ and the transmission of hybrid offspring can utilize new vectors in their circulation $[17,18]$.

\section{Methods}

\section{Leishmania parasites and sand fly colonies}

Leishmania transfection by GFP and RFP plasmids was adopted from our previous work [19] and for hybrid ( $L$. major ( L. turanica) visualization we applied the method previously used for detection of $L$. donovani hybrids in sand flies co-infected with a mixture of two parental GFP/RFP expressing strains [20]. These hybrid cells simultaneously emit at GFP and RFP wavelengths and showed a patched appearance of red and green fluorescence under the microscope [20]. L. major WHOM/ IR/-/173-DsRED $\left(\mathrm{RFP}^{+}\right)$and L. turanica MRHO/MN/08/ $\mathrm{BZ18}\left(\mathrm{GFP}^{+}\right)$were maintained at $23^{\circ} \mathrm{C}$ on RPMI-1640 medium and Schneider-D medium (3:1) supplemented with $10 \%$ fetal calf serum and amicin $(200 \mu \mathrm{l} / \mathrm{ml})$. For the GFP-marked L. turanica, the selection antibiotic nourseothricin $(1 \mu \mathrm{l} / \mathrm{ml})$ was added to the culture medium. Cultures were subcultured every 3 to 5 days. Prior to sand fly infections, parasites were washed by centrifugation and resuspended in saline solution. Laboratory colonies of four sand fly species, $P$. papatasi (originally from Turkey), P. arabicus (Israel), P. sergenti (Israel) and P. duboscqi (Senegal), were maintained on $50 \%$ sucrose at $25^{\circ} \mathrm{C}$ as described previously [21].

\section{Experimental infections of sand flies}

Sand fly females (5 to 8 days old) were fed through a chick skin membrane on heat-inactivated rabbit blood (Bioveta, Ivanovice) containing 4 to 5 day old Leishmania promastigotes. The cell density (promastigotes/ $\mathrm{ml}$ ) for all L. turanica single infections in three different sand fly species (114 specimens of $P$. papatasi, $128 P$. arabicus and $78 \mathrm{P}$. sergenti) was $1 \times 10^{6}$; the same cell density was used for single infections of 68 specimens of $P$. papatasi with $L$. major. Three different cell densities were used for mixed infections of $L$. major and L. turanica in 114, 119 and 61 specimens of P. papatasi, respectively: $1 \times 10^{5}$ (combining $5 \times 10^{4}$ L. major $+5 \times 10^{4}$ L. turanica), $1 \times 10^{6}\left(5 \times 10^{5}\right.$ L. major $+5 \times 10^{5}$ L. turanica), and $1 \times 10^{7}\left(5 \times 10^{6}\right.$ L. major $+5 \times 10^{6}$ L. turanica). Bloodengorged sand fly females were separated and maintained as described above, and dissected 2, 5, 9, and 12 days post infection (DPI). The localization and intensity of infection (number of promastigotes) in the midgut were estimated under a fluorescence microscope (Olympus BX51 with an Olympus DP 70 camera). The intensity of infection was graded as being light $(<100$ parasites/gut), moderate (100-1000 parasites/gut), or heavy (> 1000 parasites/gut) as described previously $[22,23]$. Parasite location was categorized as being in the abdominal midgut (AMG), thoracic midgut (TMG), cardia region (CAR), or colonized stomodeal valve (SV-C). Each experiment was repeated at least twice. The total number of dissected sand flies in various experiments is indicated above the bars.

In addition, we searched for putative L. major/L. turanica hybrids. As $L$. donovani hybrids were previously reported as early as on day 2 [20], groups of 30-50 females were dissected into physiological solution at different DPI and the midgut homogenate was filtered using a $30 \mu \mathrm{m}$ filter (Partec) into PBS buffer. To maximize the chance of identifying hybrids, two series of experiments differing in sand fly species and flow cytometer type were carried out. Both cytometers were calibrated using positive controls $\left(\mathrm{GFP}^{+}\right.$ and $\mathrm{RFP}^{+}$transfected Leishmania line) and a negative control (wild type Leishmania strain).

In the first series of experiments, Leishmania from P. papatasi midguts were sorted by a FACS Vantage SE cell sorter with laser emitting at $488 \mathrm{~nm}$ wavelength and detection of emission at 530/30 $\mathrm{nm}\left(\mathrm{GFP}^{+}\right)$and 585/ $42 \mathrm{~nm}\left(\mathrm{RFP}^{+}\right)$. Sorting was performed in two steps: in the first step all GFP positive events were separated; then, from this presorted material all double positive events were put into a glycerol drop containing $1 \%$ formaldehyde. Since the sorter is not designed for optimal excitation of 


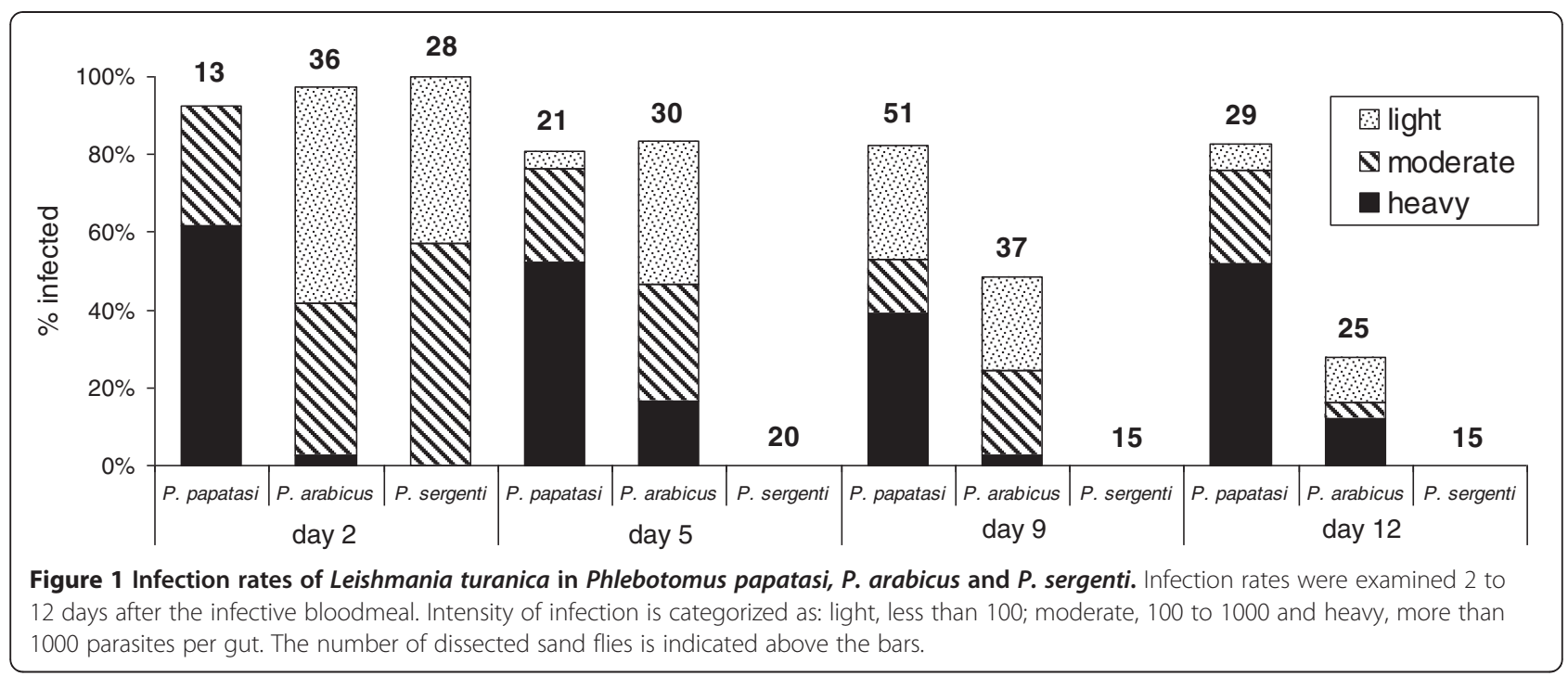

$\mathrm{RFP}^{+}$events, the total number of $\mathrm{RFP}^{+}$events was determined by an LSRII flow cytometer (BD Biosciences: excitation with the $561 \mathrm{~nm}$ laser, emission detection at $585 / 15 \mathrm{~nm}$ ).

In the second series of experiments, $P$. (Phlebotomus) duboscqi, another natural vector of $L$. major, was used. Leishmania from $P$. duboscqi midguts were sorted by a BD Influx cytometer equipped by (i) laser emitting at $488 \mathrm{~nm}$ wavelengths and detection of emission at 530/ $40 \mathrm{~nm}$ (GFP) and (ii) laser emitting at $561 \mathrm{~nm}$ wavelengths and detection of emission at 585/29 nm (RFP). These parameters allowed the separation of double positive events in one step (and without the additional use of the LSRII cytometer).

Sorted material from both series was immediately checked visually on a confocal microscope (Olympus FV1000) to distinguish and exclude sorted artifacts from potential hybrids expressing both green and red signals.

\section{Statistical analysis of experimental infections}

The software STATISTICA and R 2.13.1 (R Development Core Team [24]) were used for data analyzes. In single infections, the $\chi^{2}$ test was used to compare infection intensities (heavy, moderate, light, zero) and localizations (AMG, TMG, CAR, SV-C).

In mixed infections, the permutation-based approach [25] was used to assess differences in the proliferation pattern between L. turanica and L. major in P. papatasi. In particular, the relative prevalence of both Leishmania species within each infected sand fly was scored using a three level scale: -1 , intensity of $L$. turanica $<L$. major; 0 , intensity of $L$. turanica $\approx L$. major, and +1 , intensity of $L$. turanica $>L$. major. The null hypothesis that infection intensities do not differ between both Leishmania species cannot be excluded if confidence intervals of the mean of relative prevalence scores overlap with zero. Hence to obtain

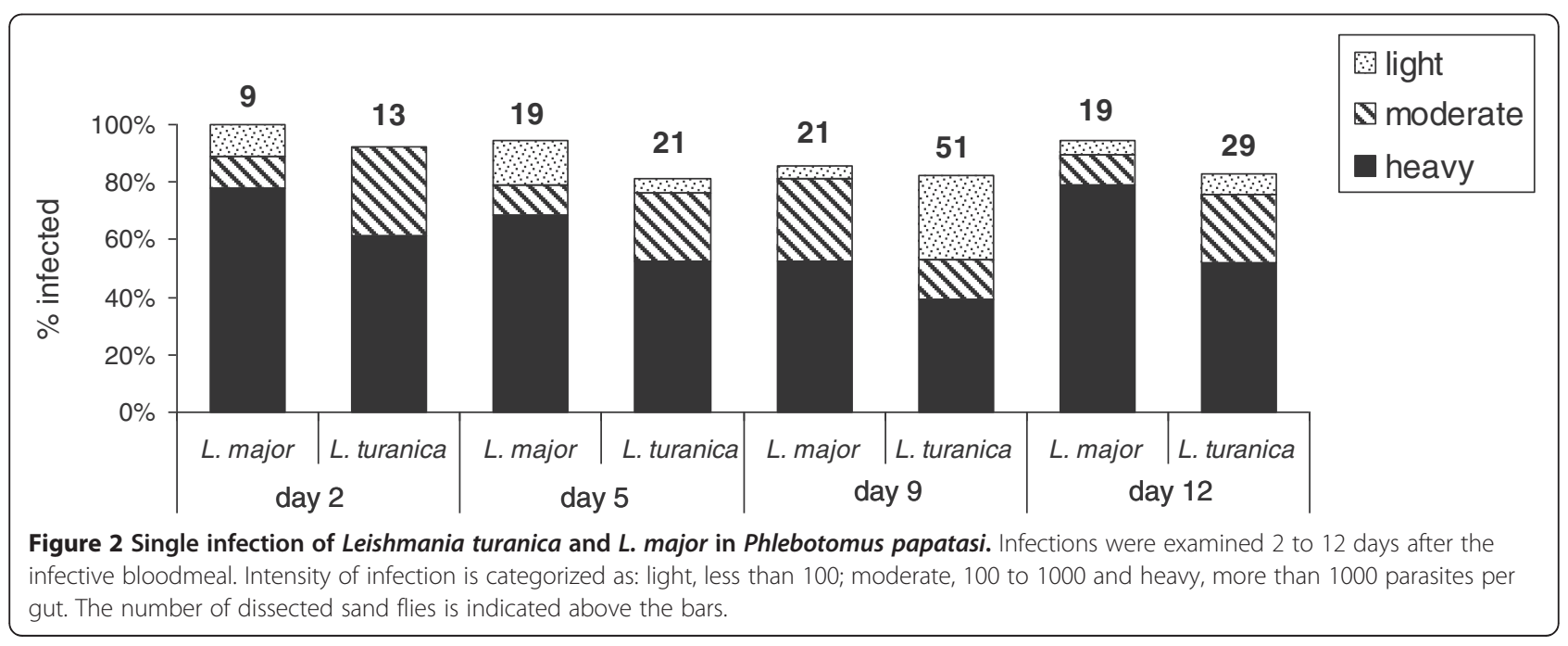


95\% confidence intervals of relative prevalence scores we bootstrapped them 1000 times. In the next step, three separate permutation based tests were performed for different initial infection doses, to evaluate the consistency of results across different initial conditions.

In the next step we evaluated the spatio-temporal dynamics of each Leishmania species infection in the digestive tract of sand flies. Two separate ANOVA models for L. turanica and L. major data were fitted to test for the relationship between the localization of infection in a particular part of the digestive tract and days after the infective feed (DPI). We further included the interaction between the localization of infection and initial infection dose, to test for the variation caused by this factor. In the next step, we scored the relative spatial distribution of both Leishmania species using the following three level scale: $-1, L$. turanica present in a more distal part of the digestive tract than L. major; 0, both Leishmania species present in the same part of the digestive tract; and $+1, L$. turanica present in a more proximal part of the digestive tract than L. major. The null hypothesis that the mean of these scores does not differ from zero was tested using bootstrap estimates of confidence intervals as described above.

\section{Results}

Comparing the development of $L$. turanica in three sand fly species

Infections of L. turanica differed significantly between all tested sand fly vector species (Figure 1): P. papatasi

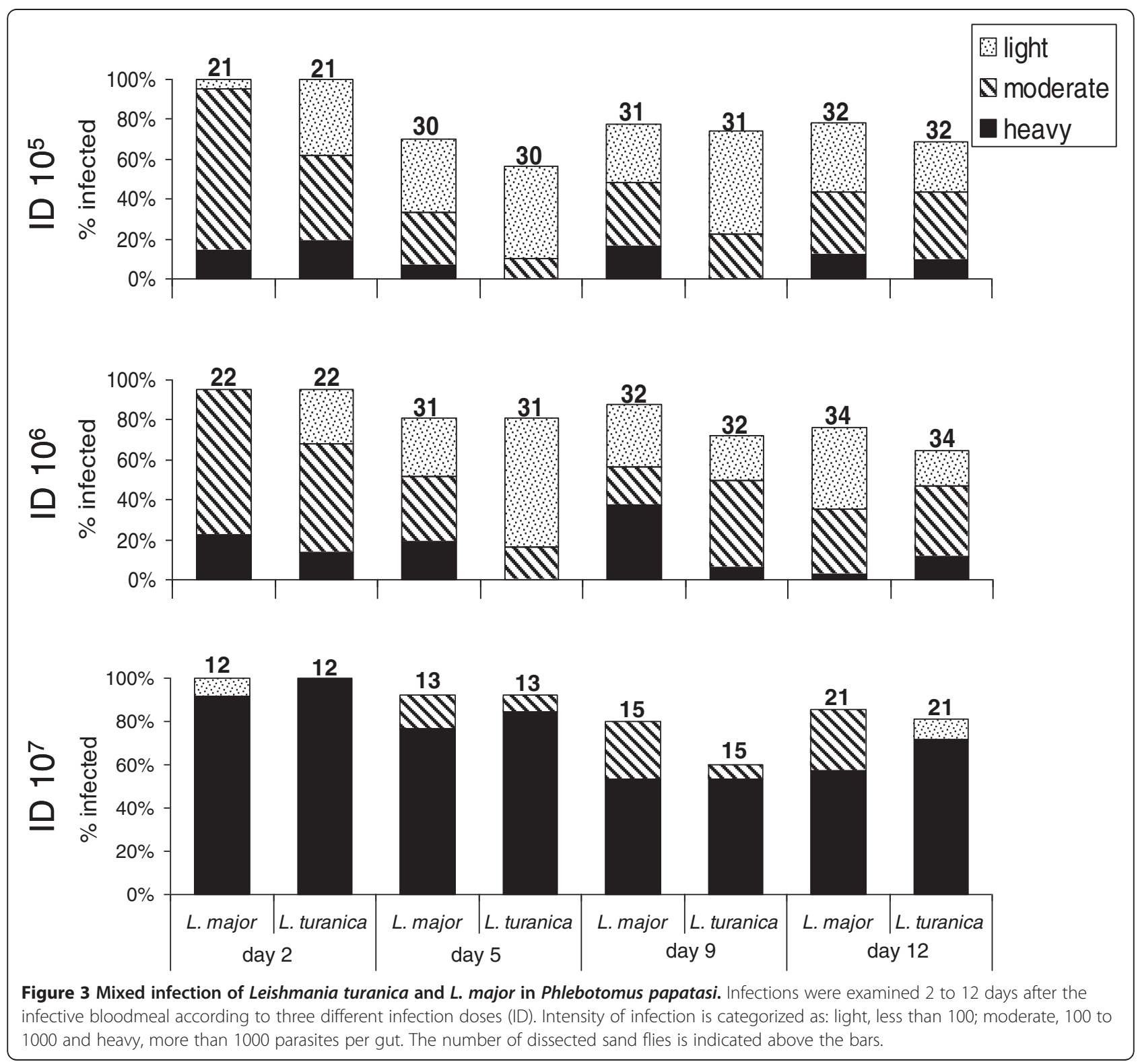


vs. $P$. arabicus (Pearson Chi-square: 50.62, $\mathrm{df}=3$, $\mathrm{p}<0.001$ ); P. papatasi vs. P. sergenti (Pearson Chisquare: $65.95, \mathrm{df}=3, \mathrm{p}<0.001) ; P$. arabicus vs. $P$. sergenti (Pearson Chi-square: 22.52, $\mathrm{df}=3, \mathrm{p}<0.001$ ).

On day 2 PI, midgut infection rates were high ( 90\%) in all studied sand fly vector species and parasites were located only in the blood meal in the AMG. On day 5 PI, when the bloodmeal was digested and remnants defecated in all three species, infection rates remained high $(\sim 80 \%)$ in $P$. papatasi and P. arabicus, but no Leishmania were found in $P$. sergenti. In $P$. papatasi most infections were heavy or moderate, whereas significantly weaker infections occurred in P. arabicus (Pearson Chisquare: $10.58, \mathrm{df}=3, \mathrm{p}=0.01$ ) (Figure 1 ).

On days 9 and $12 \mathrm{PI}$, the difference in infection rates of $P$. papatasi and P. arabicus became more marked ( $>80 \%$ in $P$. papatasi but only 48 and $28 \%$ in $P$. arabicus); in both species, however, parasites migrated anteriorly to colonize the thoracic midgut and stomodeal valve. Again, no parasites were found in P. sergenti (Figure 1).

\section{Comparing single infections of $L$. turanica and $L$. major in P. papatasi}

Leishmania turanica and L. major single infections gave high infection rates $(\sim 80 \%)$ on all examined DPI (Figure 2); however, the intensity of the L. major infection was significantly higher than that of $L$. turanica (Pearson Chi-square: 8.81, $\mathrm{df}=3, \mathrm{p}=0.03$ ). Differences in the gut locations between the L. turanica and L. major infections were insignificant on all examined DPI (Pearson Chi-square: $0.54, \mathrm{df}=3, \mathrm{p}=0.91$ ). On day $2 \mathrm{PI}$, all parasites were in the residual bloodmeal in the abdominal midgut. By day $5 \mathrm{PI}$, when the bloodmeal had been digested and remnants defecated, parasites had migrated anteriorly to the thoracic midgut and cardia region ( $L$. turanica $64 \%$; L. major 78\%), but colonization of the stomodeal valve was observed in only one female infected with $L$. major. On day $9 \mathrm{PI}$, an intense infection with colonization of the stomodeal valve developed in $50 \%$ of L. major and $48 \% \mathrm{~L}$. turanica infections; on day $12 \mathrm{PI}$, colonization of the stomodeal valve was found in $78 \% \mathrm{~L}$. major and $46 \%$ L. turanica.

\section{The development of mixed infections of L. turanica / L. major in $P$. papatasi}

The intensity of $L$. turanica and $L$. major infections was similar in the majority of $P$. papatasi females $(55.4 \%$ cases). Leishmania major overgrew L. turanica in $30.9 \%$ cases, while the opposite situation was observed in $13.7 \%$ of individuals. Three different doses of $L$. turanica/L. major mixed infections in $P$. papatasi were analyzed separately (see Figure 3). Bootstrap estimates of 95\% CI for relative prevalence scores do not overlap with zero $($ Mean $=-0.171,95 \%$ CI range $=-0.248 \sim-0.121, \mathrm{p}<$
0.001), suggesting that L. major is in general slightly more prevalent that L. turanica. However, the effect size of this difference was relatively low: the tendency for $L$. major infections to have higher intensities was revealed in groups that were exposed to $10^{5}$ and $10^{6}$ initial infection doses (Mean $=-0.262,95 \%$ CI range $=-0.377 \sim-0.149, \mathrm{p}<0.001$ and Mean $=-0.212,95 \%$ CI range $=-0.327 \sim-0.092$, $\mathrm{p}<0.001$, respectively), yet we did not find any difference between $L$. turanica and $L$. major when initial infection doses were $10^{7}$ (Mean $=+0.045$, 95\% CI range $=-0.092 \sim$ $+0.184, \mathrm{p}=0.719$ ). Significant variation of relative prevalence scores for $L$. turanica vs. $L$ major according to initial infection doses was detected using the chi square test $(\mathrm{df}=4$, $x^{2}=11.04, p=0.026$ ). (Figure 3 ).

The interaction between initial infection dose and infection localization was not significant in $L$. major $\left(\mathrm{F}_{(6,237)}=1.702, \mathrm{p}=0.121\right)$, yet this interaction was marginally significant in L. turanica $\left(\mathrm{F}_{(6,216)}=2.17, \mathrm{p}=0.047\right)$. Aside from this interactive effect, both strains exhibited a strong relationship between the infection localization and DPI (One-way ANOVA: $\mathrm{F}_{(3,245)}=100.15, \mathrm{p}<0.001$ and $\mathrm{F}_{(3,224)}=103.39, \mathrm{p}<0.001$ for $L$. major and L. turanica respectively). This pattern was comparable for both Leishmania species (Figure 4), and differences between L. turanica and L. major in the time-dependent colonization of various sand fly gut sections were nonsignificant. Leishmania major and L. turanica were present in the same part of the digestive tract in the vast majority of dissected individuals (182 cases, 82.7\%). The number of cases in which L. major or L. turanica were localized in more proximal parts of the digestive

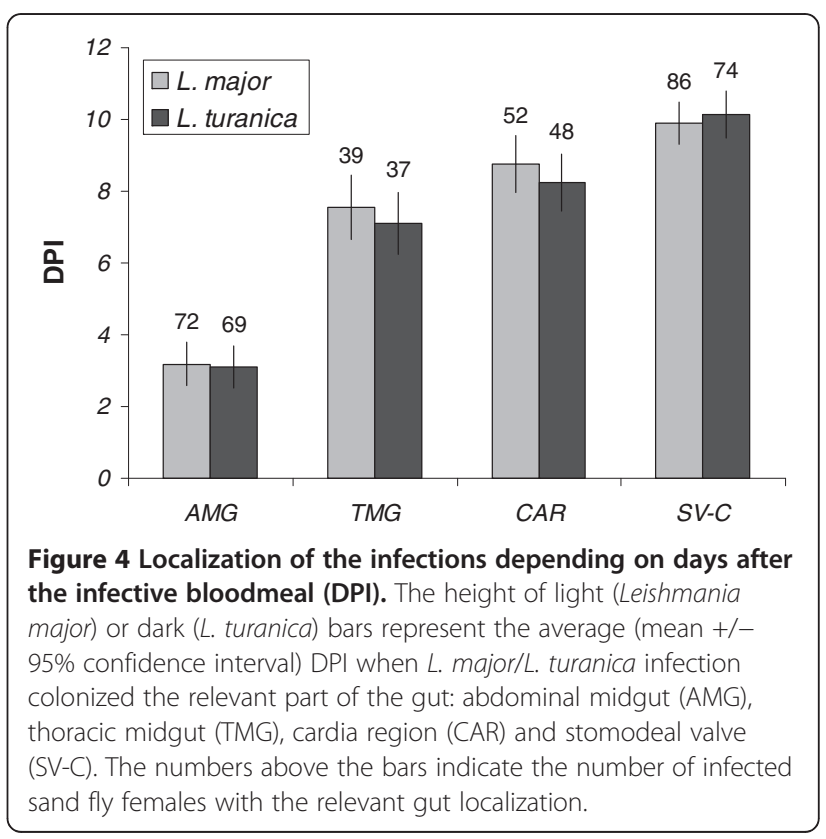


tract than the other strain were comparable (17 cases, $7.7 \%$ and 21 cases, 9.5\% respectively). Bootstrap based, 95\% confidence intervals for relative position scores of both strains in the digestive tract overlapped with zero (95\% CI range: $-0.03634 \sim+0.0545, \mathrm{p}=0.244$ ). Furthermore, no deviation from the null hypothesis expectation was detected when performing this test separately for individual initial infection doses. Finally, the relative localization of the infection did not vary significantly according to initial infection doses as demonstrated using the chi square test $\left(\mathrm{df}=4, \mathrm{x}^{2}=1.64, \mathrm{p}=0.802\right)$. This indicates that there is no pronounced difference in the spatio-temporal dynamic of colonization of the digestive tract between both Leishmania species. The development of L. turanica and L. major mixed infections and localization in $P$. papatasi midguts is illustrated in Figure 5.

The attempt to detect $L$. turanica and $L$. major hybrids was not successful. In the first series of experiments, a total of 100 co-infected females of $P$. papatasi were dissected on 2 DPI and 80 females on 9 DPI. Putative hybrid cells were sorted by a FACS Vantage SE cell sorter and observed by a confocal fluorescence microscope. For example, 31 putative hybrids were sorted at 2 DPI
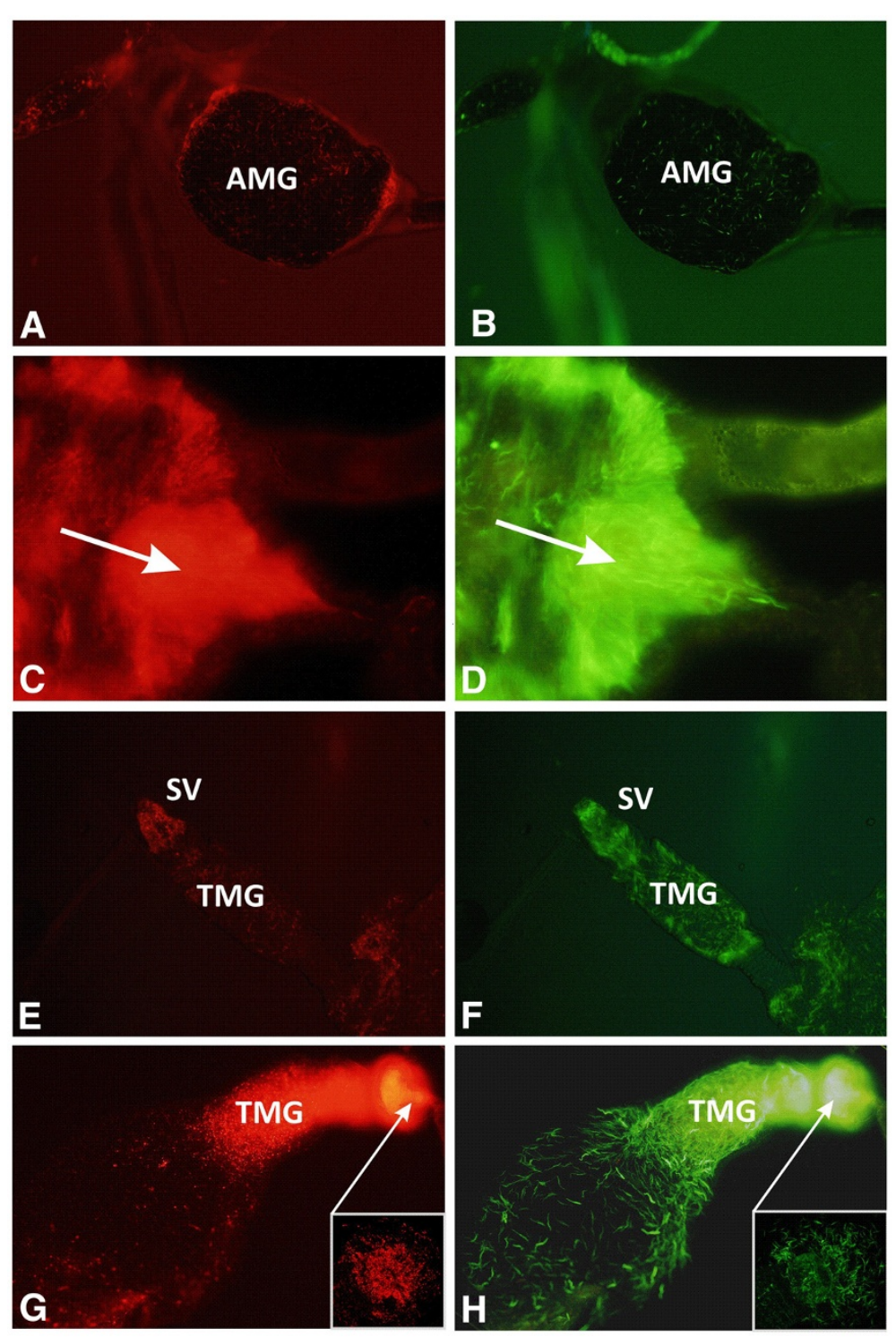

Figure 5 Development and localization of mixed Leishmania turanica and L. major infections in P. papatasi guts. L. turanica was transfected by GFP (B, D, F, H) while L. major was transfected by RFP (A, C, E, G). In the early stage of infection (2 DPI) parasites multiply in the bloodmeal in the abdominal midgut, AMG (A, B). After defecation (5 DPI) parasites reach the apical part of the AMG (arrow) (C, D), while colonization of the thoracic midgut (TMG) and stomodeal valve (SV) is typical for late infections (9 DPI) (E, F). On day 12 DPI, a heavy infection of the colonized SV (arrow) is prevalent $(\mathbf{G}, \mathbf{H})$. 
(see Figure 6); however, all were found to be artifacts (sand fly gut remnants). In the second series of experiments, a total of 130 co-infected $P$. duboscqi females were dissected on days 2, 9 and 14 PI. Samples were analyzed by a BD Influx cell sorter but again no hybrids were confirmed by fluorescent microscopy.

\section{Discussion}

This study provides experimental evidence that L. turanica develops late-stage infections in $P$. papatasi. According to the criteria of Killick-Kendrick [26], this finding, together with the well-known anthropophily of this sand fly species and repeated isolation of L. turanica from wild-caught $P$. papatasi in Central Asia (reviewed by [3]) and Iran $[9,10]$, incriminate $P$. papatasi as the vector of $L$. turanica.

Comparing the development of L. turanica and $L$. major in P. papatasi revealed high similarity and found that statistical differences are not significant from a biological point of view. Although the differences in intensities between L. turanica and L. major infections were statistically significant $(\mathrm{p}=0.03)$, the rates of infections were at the same high level in both species (around 80\% of analyzed females were positive on all examined days) and the development of location and stomodeal valve colonization ability were the same in both species without significant differences.

The ability of $L$. major to develop in the specific (selective) vector $P$. papatasi depends on the presence of species-specific modifications of the major surface

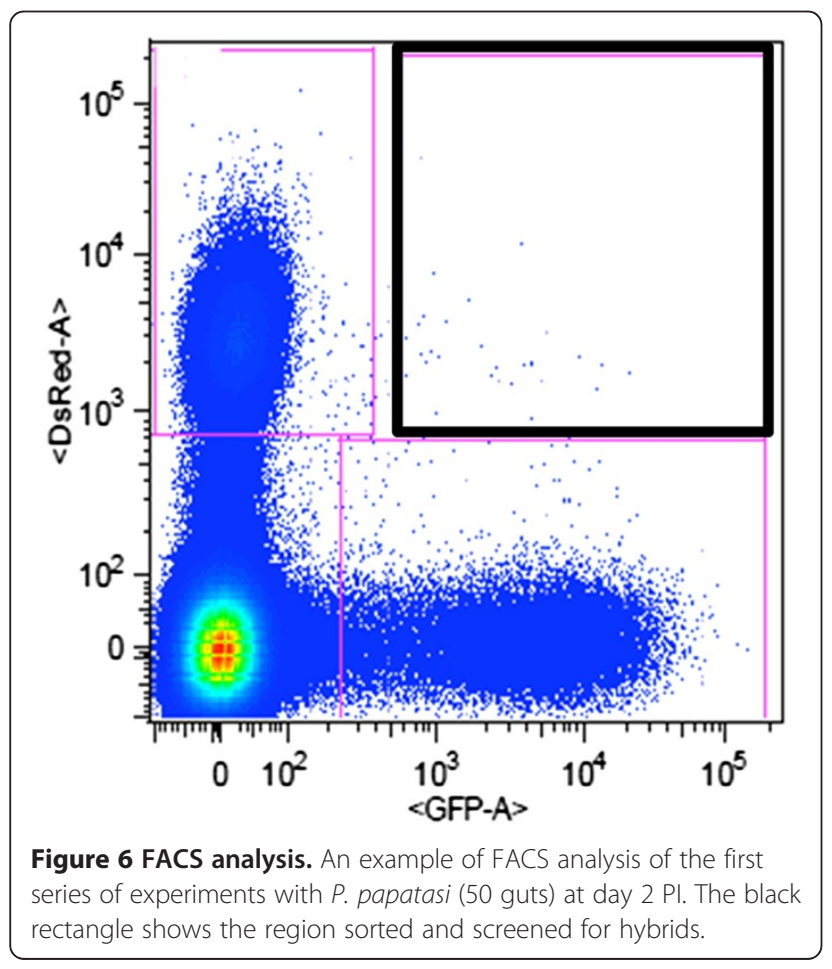

glycoconjugate of promastigotes, lipophosphoglycan (LPG), which controls the parasite attachment to the sand fly gut $[12,27]$. The successful development of $L$. turanica in $P$. papatasi suggests that the LPG of this species must be identical or very similar to that of $L$. major.

Experiments on the development of L. turanica in two other sand flies distributed in the Middle East revealed that $L$. turanica develop late stage infections in $P$. (Adlerius) arabicus but not in P. (Paraphlebotomus) sergenti. In all P. sergenti examined, infection did not persist after digestion and defecation of the bloodmeal. This result is in agreement with previous findings that $P$. arabicus is a permissive vector susceptible to various Leishmania species, including $L$. major [23,28], while $P$. sergenti is the specific vector of $L$. tropica and is refractory to L. major $[29,30]$. Reports of L. turanica from other Paraphlebotomus species [1,9], however, suggest that vector competence for Leishmania may differ between members of this subgenus.

Using GFP and RFP-marked parasites we have experimentally demonstrated that L. turanica and L. major are able to develop in P. papatasi together, without any visible sign of competition. First, we used an initial infection dose of $1 \times 10^{7}$ (resp. $5 \times 10^{6}$ L. major $+5 \times 10^{6}$ L. turanica) promastigotes $/ \mathrm{ml}$. Parasites did not differ in intensities or localization on all examined days, and the infections developed well with high rates of heavy infections for both species. Next, we decided to decrease the infection dose to better simulate natural conditions. Again, no significant differences were observed in the localization of infections and both Leishmania species colonized the stomodeal valve during mixed infections. Minor differences found in the infection intensities seem to be biologically unimportant, as other parameters, like colonization of the stomodeal valve, are more important for the vector status of the sand fly than the exact parasite number found in the midgut [23].

Despite the fact that both Leishmania species coexisted in the $P$. papatasi and $P$. duboscqi midgut for the relatively long period of two weeks (until the end of the experiment), the presence of dual fluorescing L. major / L. turanica hybrids was not demonstrated. Sadlova et al. [20] speculate that there may be species-specific differences among Leishmania species in their capacity for sexual reproduction and it should be noted that although $L$. turanica and $L$. major belong to the subgenus Leishmania, they are not closely related to each other [31]. Considering this, the similarity of LPG in L. turanica and L. major proposed here is likely to be a result of co-evolutionary convergence due to the adaptation of these two Leishmania species to the same sand fly vector.

\section{Competing interests}

The authors declare that they have no competing interests. 


\section{Authors' contributions}

ACH carried out the laboratory work of parasite development, drafted the first version of the manuscript and contributed to data analysis. JS performed data analysis of mixed infections. KK and VS carried out the detection of putative leishmania hybrids. JK performed the statistical analysis of data. MJ prepared GFP transfected L. turanica. ChS, SG and YM provided L. turanica strain. JV and PV designed the study, contributed to interpretation and finalized the manuscript. All authors read and approved the final version of the manuscript.

\section{Acknowledgements}

This study was partially funded by the Czech Ministry of Education (MSM 0021620828) and by the EU grant 2011-261504 EDENEXT. The paper is catalogued by the EDENEXT Steering Committee as EDENext067 (http:// www.edenext.eu).

\section{Author details}

'Department of Parasitology, Fac. Sci, Charles University in Prague, Prague, Czech Republic. ${ }^{2}$ Department of Zoology, Fac. Sci, Charles University in Prague, Prague, Czech Republic. ${ }^{3}$ Biology Centre, Institute of Parasitology, Ceske Budejovice, Czech Republic. ${ }^{4}$ Department of Molecular Immunology, University of Tokyo, Tokyo, Japan.

Received: 17 July 2012 Accepted: 14 September 2012

Published: 2 October 2012

\section{References}

1. Guan $L R$, Yang $Y Q, Q u J Q$, Shen WX: Discovery and study of Leishmania turanica for the first time in China. B World Health Organ 1995, 73:667-672.

2. Gramiccia M, Gradoni L: The current status of zoonotic leishmaniases and approaches to disease control. Int J Parasitol 2005, 35:1 169-1180.

3. Strelkova MV: Progress in studies on Central Asian foci of zoonotic cutaneous leishmaniasis: a review. Folia Parasit 1996, 43:1-6.

4. Strelkova MV, Shurkhal AV, Kellina Ol, Eliseev LN, Evans V, Peters W, Chapman CJ, Le Blancq SM, van Eys GJJM: New species of Leishmania isolated from the great gerbil Rhombomys opimus. Parasitology 1990, 101:327-335.

5. Strelkova MV, Eliseev LN, Ponirovsky EN, Dergacheva TI, Annacharyeva DK, Erokhin PI, Evans DA: Mixed leishmanial infections in Rhombomys opimus: a key to the persistence of Leishmania major from one transmission season to the next. Ann Trop Med Parasit 2001, 95:811-819.

6. Akhavan AA, Mirhendi H, Khamesipour A, Alimohammadian MH, Rassi Y, Bates P, Kamhawi S, Valenzuela JG, Arandian MH, Abdoli H, Jalali-zand N, Jafari R, Shareghi N, Ghanei M, Yaghoobi-Ershadi MR: Leishmania species: detection and identification by nested PCR assay from skin samples of rodent reservoirs. Exp Parasitol 2010, 126:552-556.

7. Mirzaei A, Rouhani S, Taherkhani H, Farahmand M, Kazemi B, Hedayati M, Baghaei A, Davari B, Parvizi P: Isolation and detection of Leishmania species among naturally infected Rhombomys opimus, a reservoir host of zoonotic cutaneous leishmaniasis in Turkemen Sahara, North East of Iran. Exp Parasitol 2011, 129:375-380.

8. Strelkova MV: Susceptibility to and the characteristics of the course of experimental leishmaniasis in different species of mammals infected with Leishmania major, L. turanica and L. gerbilli. Med Parasitol Parasit Dis 1991, 1:35-39. In Russian.

9. Parvizi P, Ready PD: Nested PCRs of nuclear ITS-rDNA fragments detect three Leishmania species of gerbils in sanflies from Iranian foci of zoonotic cutaneous leishmaniasis. Trop Med Int Health 2008, 13:1 159-1171.

10. Rassi Y, Oshaghi MA, Azani SM, Abaie MR, Rafizadeh S, Mohebai M, Mohtarami F, Zeinali M: Molecular detection of Leishmania infection due to Leishmania major and Leishmania turanica in the vectors and reservoir host in Iran. Vector-Borne Zoonotic Dis 2011, 11:145-150.

11. Strelkova MV, Shendrik AG, El Fari M, Schonian G: Ecology and the genetic structure of sympatric leishmania species circulating in the intracontinental deserts of the south Palaearctic region. Med Parasitol Parasit Dis 2003, 3:12-8. In Russian.

12. Kamhawi S: Phlebotomine sand flies and Leishmania parasites: friends or foes? Trends Parasitol 2006, 22:439-445.

13. Volf P, Myskova J: Sand flies and Leishmania: specific versus permissive vectors. Trends in Parasitol 2007, 23:91-92.
14. Miles MA, Yeo M, Mauricio IL: Leishmania exploit sex. Science 2009, 324:187-189.

15. Schwenkenbecher JM, Wirth $T$, Schnur LF, Jaffe CL, Schallig H, Al-Jawabreh A, Hamarsheh O, Azmi K, Pratlong F, Schönian G: Microsatellite analysis reveals genetic structure of Leishmania tropica. Int J Parasitol 2006 36:237-246.

16. Nolder D, Roncal N, Davies CR, Llanos-Cuentas A, Miles MA: Multiple hybrid genotypes of Leishmania (Viannia) in a focus of mucocutaneous leishmaniasis. Am J Trop Med Hyg 2007, 76:573-578.

17. Volf, Benkova I, Myskova J, Sadlova J, Campinob L, Ravelc C: Increased transmission potential of Leishmania major/Leishmania infantum hybrids. Int J Parasitol 2007, 37:589-593.

18. Volf P, Sadlova J: Sex in Leishmania. Science 2009, 324:1644

19. Lukeš J, Paris Z, Regmi S, Breitling R, Mureev S, Kushnir S, Pyatkov K, Jirků M, Alexandrov KA: Translational initiation in Leishmania tarentolae and Phytomonas serpens (Kinetoplastida) is strongly influenced by pre-ATG triplet and its $5^{\prime}$ sequence context. Mol Biochem Parasitol 2006 148:125-132.

20. Sadlova J, Yeo M, Seblova V, Lewis MD, Mauricio I, Volf P, Miles MA: Visualisation of Leishmania donovani fluorescent hybrids during early stage development in the sand fly vector. PLOS One 2011, 6:e19851.

21. Volf $P$, Volfova $V$ : Establishment and maintenance of sand fly colonies. J Vector Ecol 2011, 36:S1-S9.

22. Ciháková J, Volf P: Development of different Leishmania major strains in the vector sandflies Phlebotomus papatasi and P. duboscqi. Ann Trop Med Parasit 1997, 9:267-279.

23. Myskova J, Votypka J, Volf P: Leishmania in sand flies: comparison of quantitative polymerase chain reaction with other techniques to determine the intensity of infection. J Med Entomol 2008, 45:133-138.

24. R Development Core Team: $R$ : A language and environment for statistical computing. Vienna, Austria: R Foundation for Statistical Computing; 2011. URL http://www.R-project.org/. ISBN 3-900051-07-0.

25. Good P: Permutation tests: A practical guide to resampling methods for testing hypotheses. 2nd edition. Berlin: Springer; 2000:270pp.

26. Killick-Kendrick R: Phlebotomine vectors of the leishmaniases: a review. Med Vet Entomol 1990, 4:1-24.

27. Pimenta PF, Saraiva EM, Rowton E, Modi GB, Garraway LA, Beverley SM, Turco SJ, Sacks DL: Evidence that the vectorial competence of phlebotomine sand flies for different species of Leishmania is controlled by structural polymorphisms in the surface lipophosphoglycan. Proc Natl Acad Sci USA 1994, 91:9155-9159.

28. Svobodova M, Volf $\mathrm{P}$, Votypka J: Experimental transmission of Leishmania tropica to hyraxes (Procavia capensis) by the bite of Phlebotomus arabicus. Microbes Infect 2006, 8:1691-1694.

29. Killick-Kendrick R, Killick-Kendrick M, Tang Y: Anthroponotic cutaneous leishmaniasis in Kabul, Afghanistan: the low susceptibility of Phlebotomus papatasi to Leishmania tropica. Trans R Soc Trop Med Hyg 1994, 88:252-253.

30. Kamhawi S, Modi GB, Pimenta PF, Rowton E, Sacks DL: The vectorial competence of Phlebotomus sergenti is specific for Leishmania tropica and is controlled by species-specific, lipophosphoglycan-mediated midgut attachment. Parasitology 2000, 121:25-33.

31. Asato Y, Oshiro M, Myint CK, Yamamoto Y, Kato H, Marco JD, Mimori T, Gomez EA, Hashiguchi Y, Uezato H: Phylogenic analysis of the genus Leishmania by cytochrome b gene sequencing. Exp Parasitol 2009, 121:352-361.

doi:10.1186/1756-3305-5-219

Cite this article as: Chajbullinova et al:: The development of Leishmania turanica in sand flies and competition with L. major. Parasites \& Vectors 2012 5:219. 